\title{
Functionally Graded Tunable Microwave Absorber with Graphene-Augmented Alumina Nanofibers
}

\author{
Ali Saffar Shamshirgar ${ }^{1}$, Rocio E. Rojas Hernández ${ }^{1}$, Girish C. Tewari², José Francisco \\ Fernández ${ }^{3}$, Roman Ivanov ${ }^{1}$, Maarit Karppinen $^{2}$, Irina Hussainova ${ }^{{ }^{*}}$
}

1. Department of Mechanical and Industrial Engineering, Tallinn University of Technology, Tallinn, Estonia

* Corresponding author. Tel: +372 620 3371. E-mail: irina.hussainova@taltech.ee

2. Department of Chemistry and Materials Science, Aalto University, P.O. Box 16100, FI00076 Aalto, Finland

3. Institute of Ceramics and Glass (ICV-CSIC), Kelsen 5, 28049 Madrid, Spain 
Refractive index is the product of the square root of the $\varepsilon_{\mathrm{r}} \times \mu_{\mathrm{r}}$ and has two components of real part (n) and imaginary part (k) and correspond to refraction and absorption of the incident, respectively. The two values for composite structures of GAIN/alumina are presented in Figure S1a,b. AC conductivity represents the absorption of energy via joule heating (real part) and $\pi / 2$ out of phase inductive current (imaginary part). It can be correlated to dielectric properties via $\sigma_{\mathrm{AC}}=\omega \varepsilon_{0} \varepsilon^{\prime}$ relation. The AC conductivity of each GAIN/alumina composition is presented in Figure S1c. Skin depth $(\delta)$ of each composition is given by $(2 / \omega \mu \sigma)^{-1 / 2}$ in Figure S1d.

a
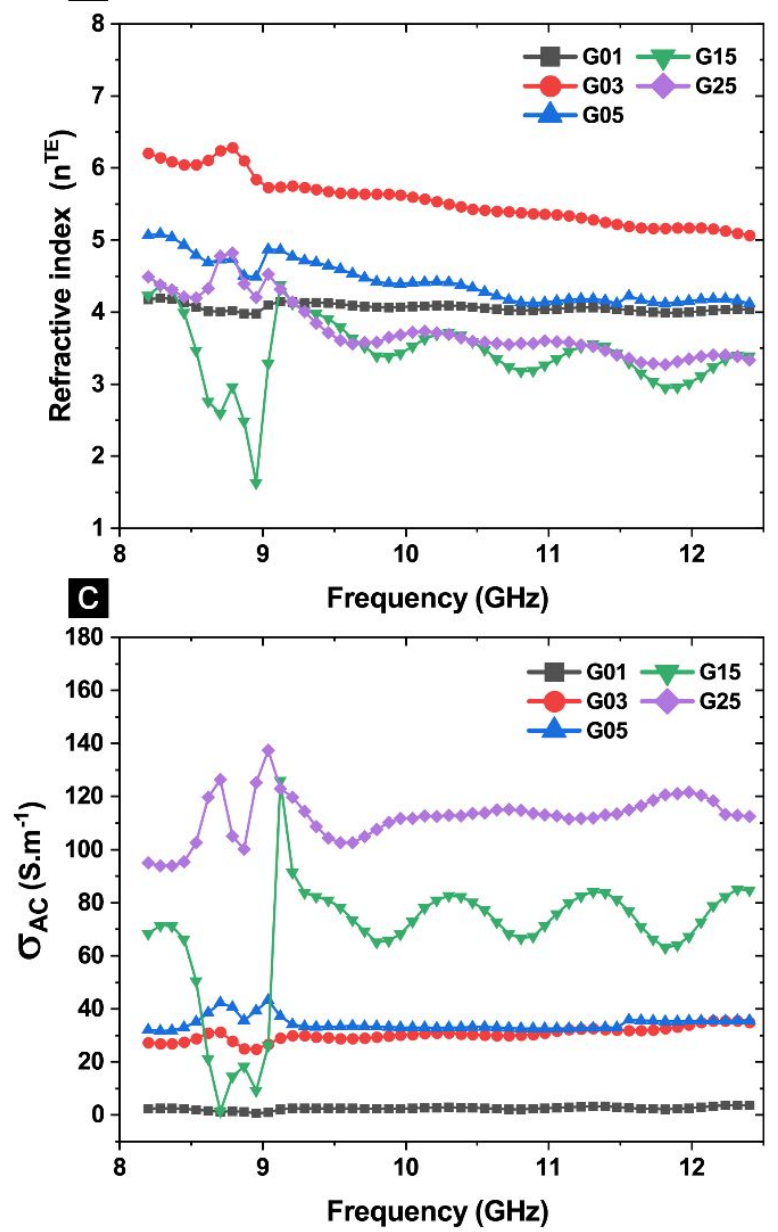
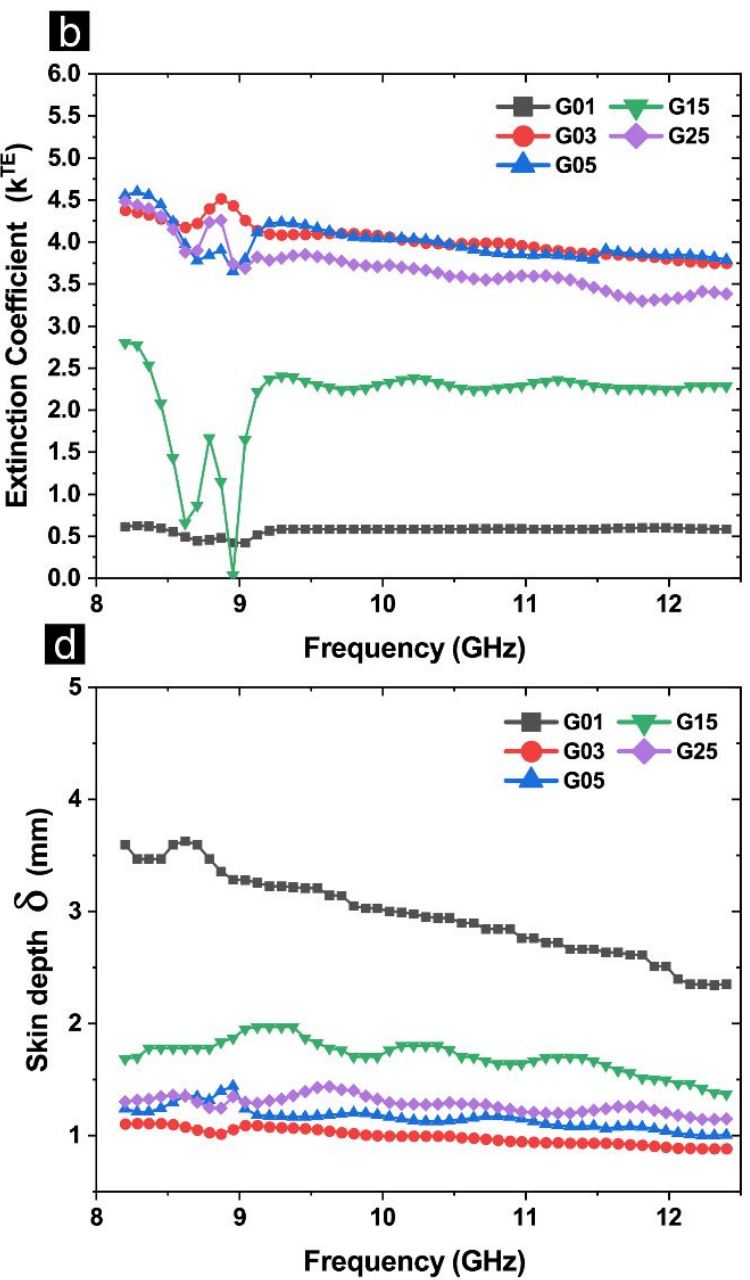

Figure S1. (a-b) Refractive index $\mathrm{n}$ and k values respectively; (c) AC conductivity of bulk composites; (d) skin depth as a function of GAIN loading in bulk composites. 
Vickers microhardness test was carried out under 4.9 N load (HV0.5) using a BUEHLER MICROMET 2001 device equipped with a square-based orthogonal pyramidal diamond indenter. Five indents were introduced on the cross section of each layers of sample FSM-891, and the mean values are reported in Figure S2a. Presence of intragranular porosity is visible in alumina layers (Figure S2b). In layer $\mathrm{h}_{6}$ were $1 \mathrm{wt} . \%$ of GAIN fillers are introduced, a refinement of the microstructure together with a lower level of porosity results in an slight increase in hardness reaching a value of $\sim 21 \mathrm{GPa}$. Following that, the hardness decreases as a function of GAIN content to a minimum of $\sim 8$ GPs in the layer with $25 \mathrm{wt} . \%$ filler content. Crack trajectory in the pristine alumina layers is intragranular and in most cases stops in a porosity. In contrast, presence of fibrous fillers promotes crack deflection and pinning effect, more noticeable in $h_{2}$ and $h_{3}$ layers with highest GAIN contents.
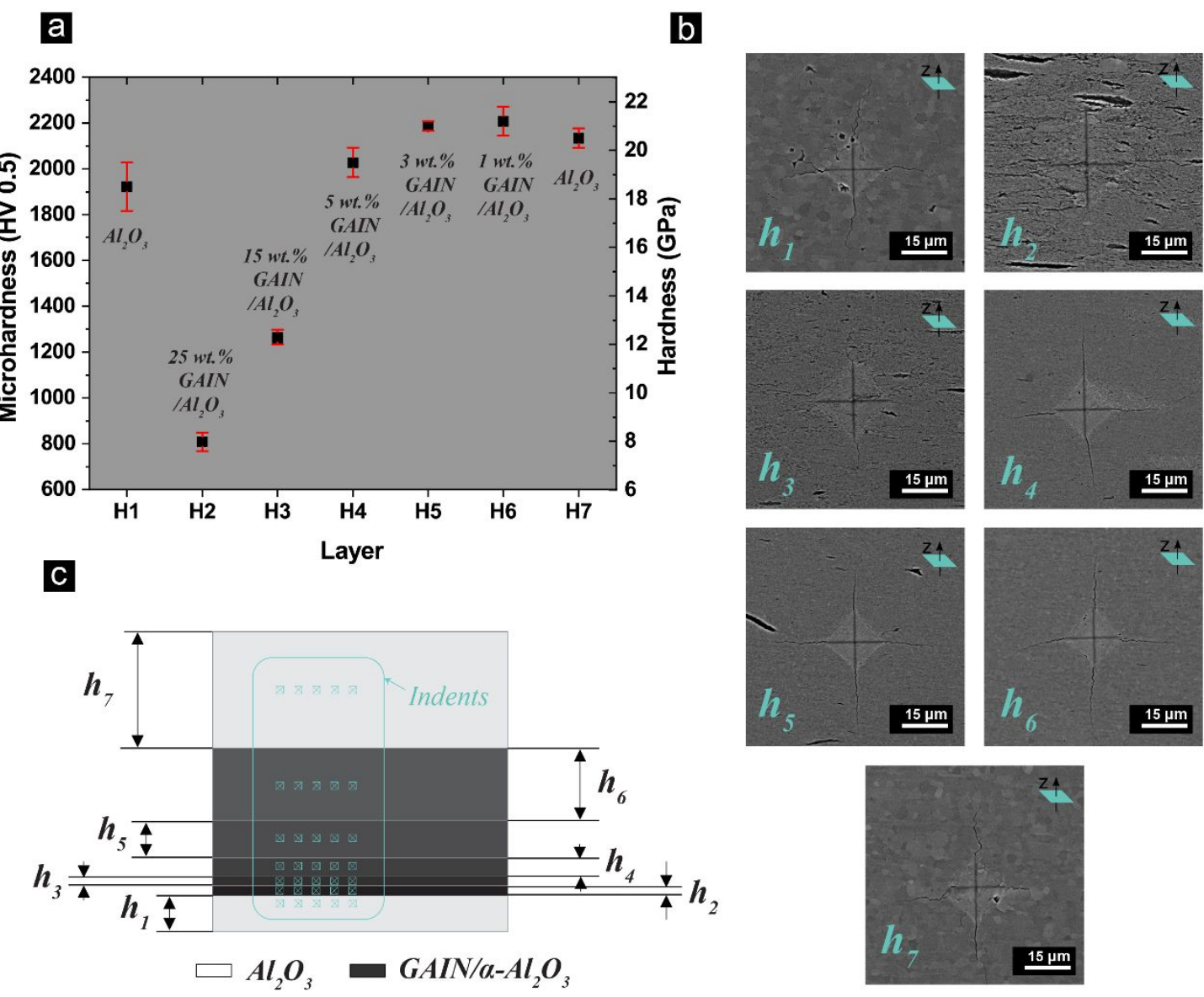

Figure S2. (a) Microhardness values for HV 0.5; (b) microhardness indentations in various layers; (c) schematic representation of the test specimen. 
A schematic of graphene augmented inorganic nanofiber (GAIN) fillers, optical and SEM images of alumina nanofibers before and after CVD, and TEM images of GAIN are shown in Figure S4a-e. A discontinuous highly defective nanocrystalline few-layer graphene with 2 to 5 layers is visible in Figure S4c. TEM images of various regions of the fibers indicate a variety of stacked graphene structures with a wide range of layer length and morphology that have stemmed from the defective surface of the $\gamma-\mathrm{Al}_{2} \mathrm{O}_{3}$ nanofibers used as the substrate Figure $\mathbf{S 4 d}$ e. Raman spectrum of GAIN in Figure S4f and deconvolution of the peaks in Figure S4g-h can help to elucidate the nature of the carbon coating.

To begin with, the high intensity of the defect modulated D peak at $1342 \mathrm{~cm}^{-1}$ as compared to $\mathrm{G}$ peak at $1585 \mathrm{~cm}^{-1}\left(\mathrm{I}_{\mathrm{D}} / \mathrm{I}_{\mathrm{G}}\right.$ ratio $\sim 1.81$, Table $\left.\mathrm{S1}\right)$ indicates very small $\mathrm{sp}^{2}$ domains ${ }^{1}$, consistent with HRTEM observations. Double resonant Raman scattering theory suggests the combinational on-site and vacancy-like defect types for $\mathrm{I}_{\mathrm{D}} / \mathrm{I}_{\mathrm{G}}$ ratio of $\sim 1.8^{2,3}$. The $2 \mathrm{D}$ peak at $2669 \mathrm{~cm}^{-1}$ is broadened (as compared to a typical Raman for single layer graphene ${ }^{4}$ ) with a FWHM of $\sim 116$ and a $\mathrm{I}_{2 \mathrm{D}} / \mathrm{I}_{\mathrm{G}}$ ratio of 0.32 . The position and FWHM of the $2 \mathrm{D}$ peak together with the existence of a notable D peak can be attributed to the presence of turbostratic carbon ${ }^{4}$. The $\mathrm{D}+\mathrm{D}^{\prime}$ peak at $\sim 2915 \mathrm{~cm}^{-1}$ is the second order of the intra-valley $\mathrm{D}^{\prime}$ peak at $\sim 1620 \mathrm{~cm}^{-1}$ and its presence can be in in response to hydrogen functionalization (employed during CVD procedure) and $\mathrm{sp}^{3}$ hybridization ${ }^{5}$. The $2 \mathrm{D}$ band is $21 \mathrm{~cm}^{-1}$ redshifted as compared to a typical single layer graphene. This redshift could have stemmed from the local strain caused by the lattice mismatch between $\gamma-\mathrm{Al}_{2} \mathrm{O}_{3}$ substrate and graphene that resulted in a decrease in phonon energies $^{6}$. Even though, the same physical mechanism is expected to redshift the G peak, the $\sim 5 \mathrm{~cm}^{-1}$ blueshift of this peak is likely to have originated from the doping effect of the substrate ${ }^{7}$ (G peak for typical single layer graphene is at $\sim 1580 \mathrm{~cm}^{-1}$ measured with similar wavelength $\left.\operatorname{laser}^{8}\right)$. In fact, both $n$ - and $p$-doping shift Fermi level away from the Dirac point and decrease the recombination probability of the charge carriers. This, results in non-adiabatic perturbation of the phonons, removing the Kohn anomaly with the outcome of an increase in phonon energy of the $\mathrm{G}$ peak and the subsequent blueshift ${ }^{9,10}$. Lastly, the defect-driven $\mathrm{D}^{*}$ and $\mathrm{D}^{* *}$ peaks at $1210 \mathrm{~cm}^{-1}$ and $1510 \mathrm{~cm}^{-1}$, respectively, can be attributed to $\mathrm{sp}^{2}$-bonded configurations of transpolyacetylene segments at grain boundaries and surfaces of CVD carbon postulated by Ferarri et al. ${ }^{11}$. The peak position, peak intensities, and FWHM for different layers of the multilayered sample FSM-89-1 are listen in the Table $\mathbf{S 2 .}$ 
a

Graphene Augmented Inorganic Nanofiber (GAIN)
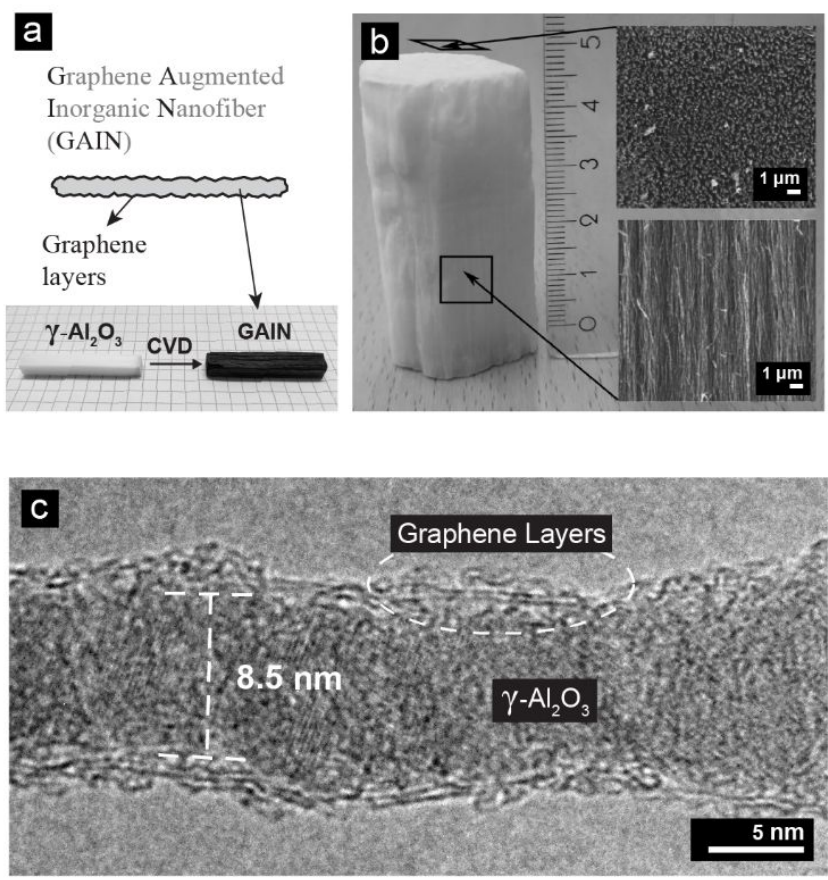

\section{g}

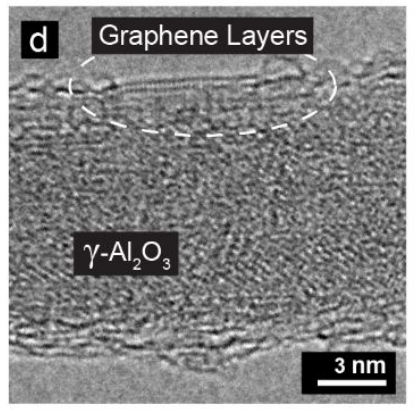

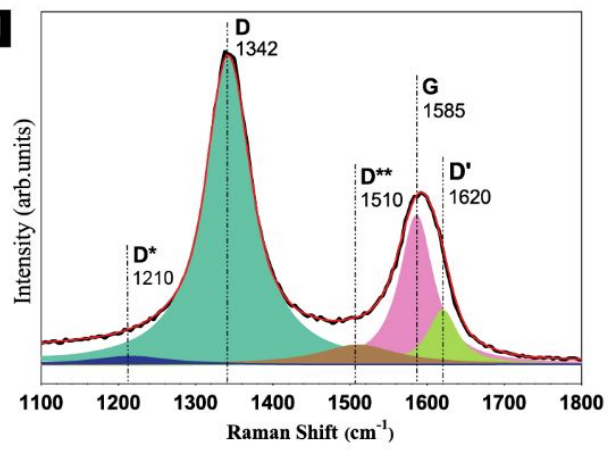

h

f
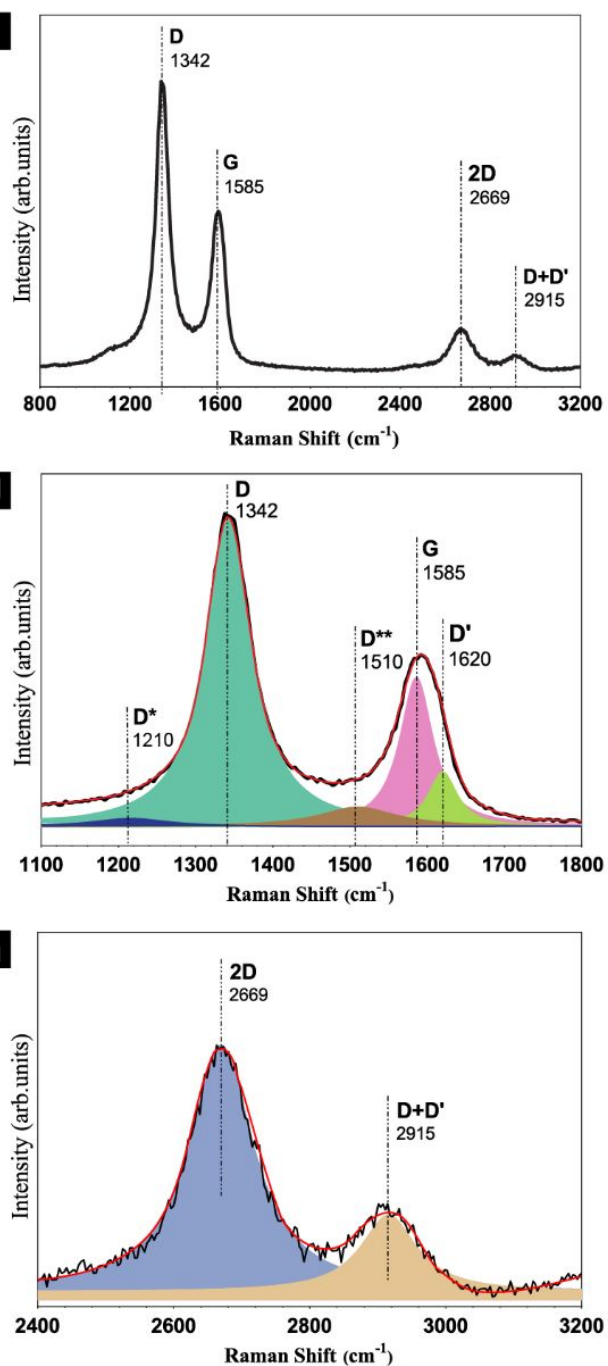

Figure S4. (a) Schematic of GAIN with $\gamma-\mathrm{Al}_{2} \mathrm{O}_{3}$ core and graphene coating together with optical images of before and after the CVD process; (b) optical image and SEM images of self-aligned $\gamma-\mathrm{Al}_{2} \mathrm{O}_{3}$ nanofibers; (c-e) HRTEM images of a GAIN fiber with 2-3 layers of graphene coating; (f) Raman spectrum of GAIN; (g) deconvolution of the peaks providing details on the Raman shift ranging from 1100 to $1800 \mathrm{~cm}^{-1}$; and (h) 2400 to $3200 \mathrm{~cm}^{-1}$.

Table S1. Peak position, FWHM, intensity ratio comparison for GAIN.

\begin{tabular}{|c|c|c|c|c|c|c|c|c|c|c|c|}
\hline \multicolumn{2}{|c|}{ D } & \multicolumn{2}{|c|}{ G } & \multicolumn{2}{|c|}{$D^{\prime}$} & \multicolumn{2}{|c|}{ 2D } & \multicolumn{2}{|c|}{$D+D^{\prime}$} & \multirow[b]{2}{*}{$\begin{array}{l}\mathrm{I}_{\mathrm{D}} / \mathrm{I}_{\mathrm{G}} \\
\text { ratio }\end{array}$} & \multirow[b]{2}{*}{$\begin{array}{l}\mathrm{I}_{2 \mathrm{D}} / \mathrm{I}_{\mathrm{G}} \\
\text { ratio }\end{array}$} \\
\hline $\begin{array}{l}\text { Peak } \\
\text { Position }\end{array}$ & FWHM & $\begin{array}{c}\text { Peak } \\
\text { Position }\end{array}$ & FWHM & $\begin{array}{c}\text { Peak } \\
\text { Position }\end{array}$ & FWHM & $\begin{array}{c}\text { Peak } \\
\text { Position }\end{array}$ & FWHM & $\begin{array}{c}\text { Peak } \\
\text { Position }\end{array}$ & FWHM & & \\
\hline 1342 & 73 & 1585 & 52 & 1620 & 45 & 2669 & 116 & 2915 & 100 & 1.81 & 0.32 \\
\hline
\end{tabular}


Table S2. Peak position, FWHM, and intensity of Raman peaks in each layer of the multilayer FSM-89-1 sample.

\begin{tabular}{|c|c|c|c|c|c|c|c|c|c|c|c|c|c|c|c|}
\hline & & D & & & G & & & $D^{\prime}$ & & & $2 \mathrm{D}$ & & & $D+D^{\prime}$ & \\
\hline Layer & $\begin{array}{c}\text { Peak } \\
\text { Position }\end{array}$ & FWHM & Intensity & $\begin{array}{c}\text { Peak } \\
\text { Position }\end{array}$ & FWHM & Intensity & $\begin{array}{c}\text { Peak } \\
\text { Position }\end{array}$ & FWHM & Intensity & $\begin{array}{c}\text { Peak } \\
\text { Position }\end{array}$ & FWHM & Intensity & $\begin{array}{c}\text { Peak } \\
\text { Position }\end{array}$ & FWHM & Intensity \\
\hline$h_{6}$ & 1359.00 & 51.67 & 188.47 & 1590.86 & 50.85 & 125.56 & 1620.75 & 43.79 & 65.41 & 2701.93 & 73.95 & 68.48 & 2945.00 & 50.94 & 4.34 \\
\hline$h_{5}$ & 1359.07 & 54.47 & 221.38 & 1590.28 & 49.48 & 135.99 & 1620.00 & 43.65 & 82.79 & 2701.43 & 79.18 & 55.71 & 2945.00 & 41.44 & 4.12 \\
\hline$h_{4}$ & 1355.12 & 53.83 & 321.21 & 1588.57 & 48.90 & 183.47 & 1620.00 & 40.00 & 107.46 & 2695.85 & 95.02 & 74.82 & 2945.74 & 58.00 & 10.81 \\
\hline$h_{3}$ & 1355.13 & 53.54 & 368.95 & 1588.57 & 49.00 & 190.35 & 1620.46 & 40.00 & 106.12 & 2692.49 & 92.12 & 73.78 & 2945.00 & 66.65 & 9.84 \\
\hline $\mathrm{h}_{2}$ & 1355.44 & 52.98 & 325.12 & 1588.57 & 49.68 & 165.78 & 1620.23 & 40.09 & 97.24 & 2692.58 & 91.29 & 65.58 & 2945.27 & 67.57 & 7.93 \\
\hline
\end{tabular}

By employing transmission line method, conversion of microwave scattering parameters (Sparameters) to complex permittivity and permeability can be done via various different approaches. The Nicholson-Ross-Weir (NRW) convert S11, S21, S12, and S22 values to both $\varepsilon$ and $\mu$. However, the method can have discontinuities for low loss samples with thickness of $>1 / 2$ wavelength. Short circuit line method (SCL) is a one-port measurement that considers a specimen backed by a reflective surface therefore no transmission can be inferred. In this approach only $\varepsilon$ is calculated and therefore is suitable for non-magnetic materials. Measured $\varepsilon$ for sample G25 using both methods are shown in Figure S5. In the measured $\varepsilon$ data for all samples, a resonance phenomenon is present in the frequency range of 8.5-9 GHz. This effect is only present in the case of GAIN/alumina composites whereas for the reference polycrystalline alumina specimen, no frequency dependence is observed (Figure S6).
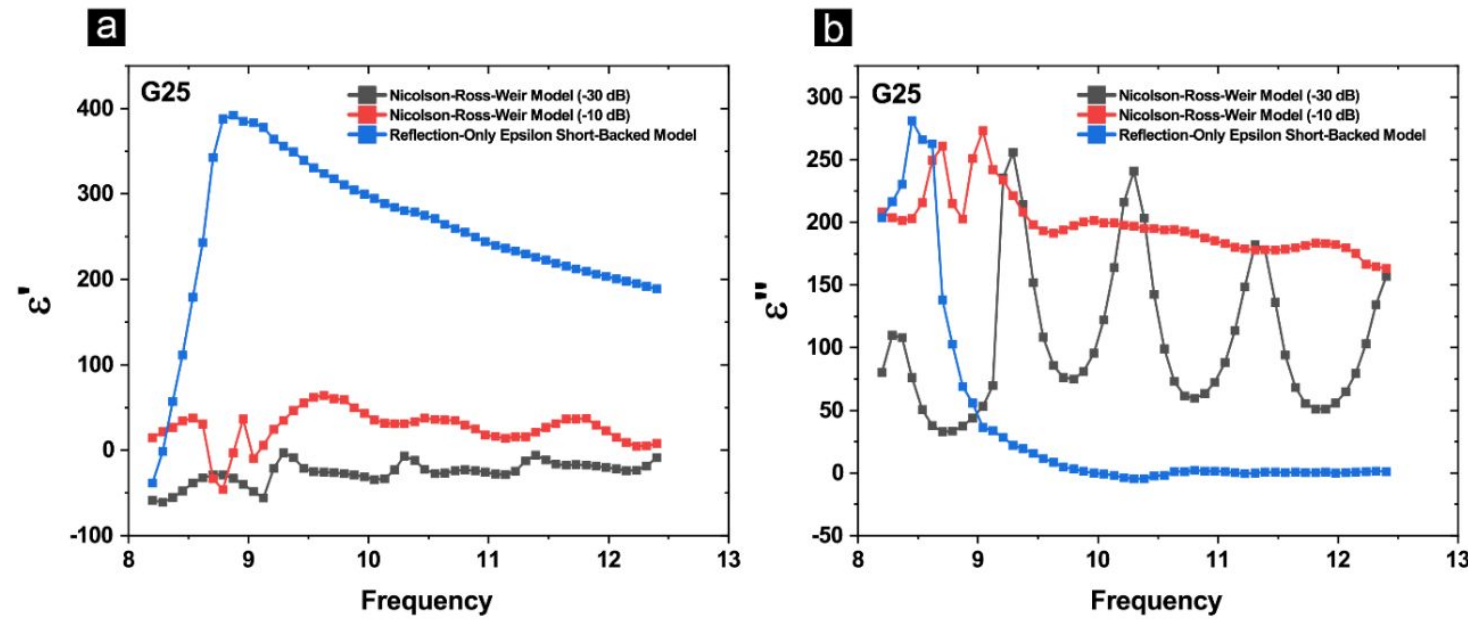

Figure S5. Various measurements of (a) real permittivity and (b) imaginary permittivity in G25 sample with 25 wt.\% of GAIN fillers. 


\section{a}

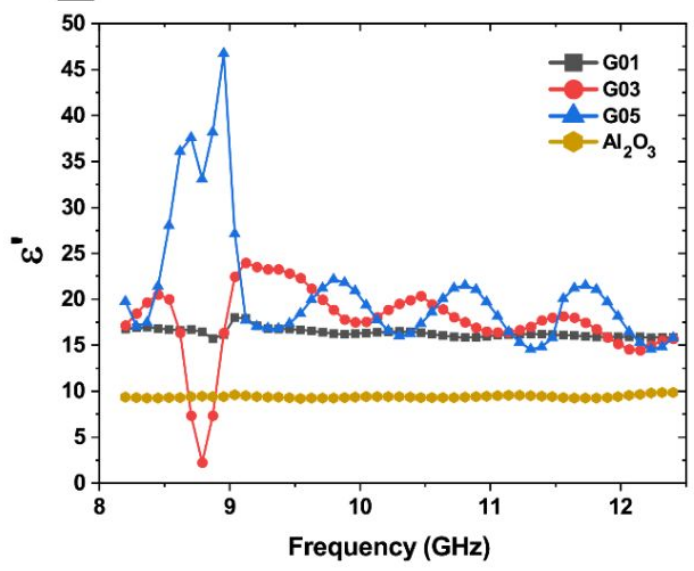

b

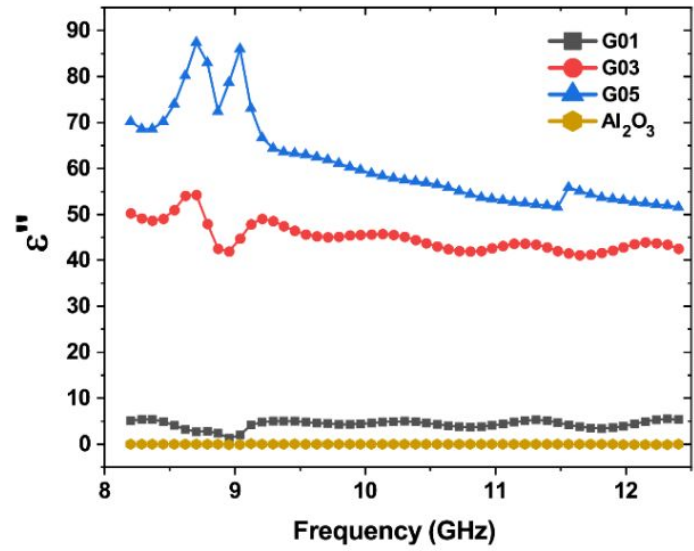

Figure S6. Measured (a) real permittivity and (b) imaginary permittivity of reference polycrystalline alumina and G01-G05 composites.
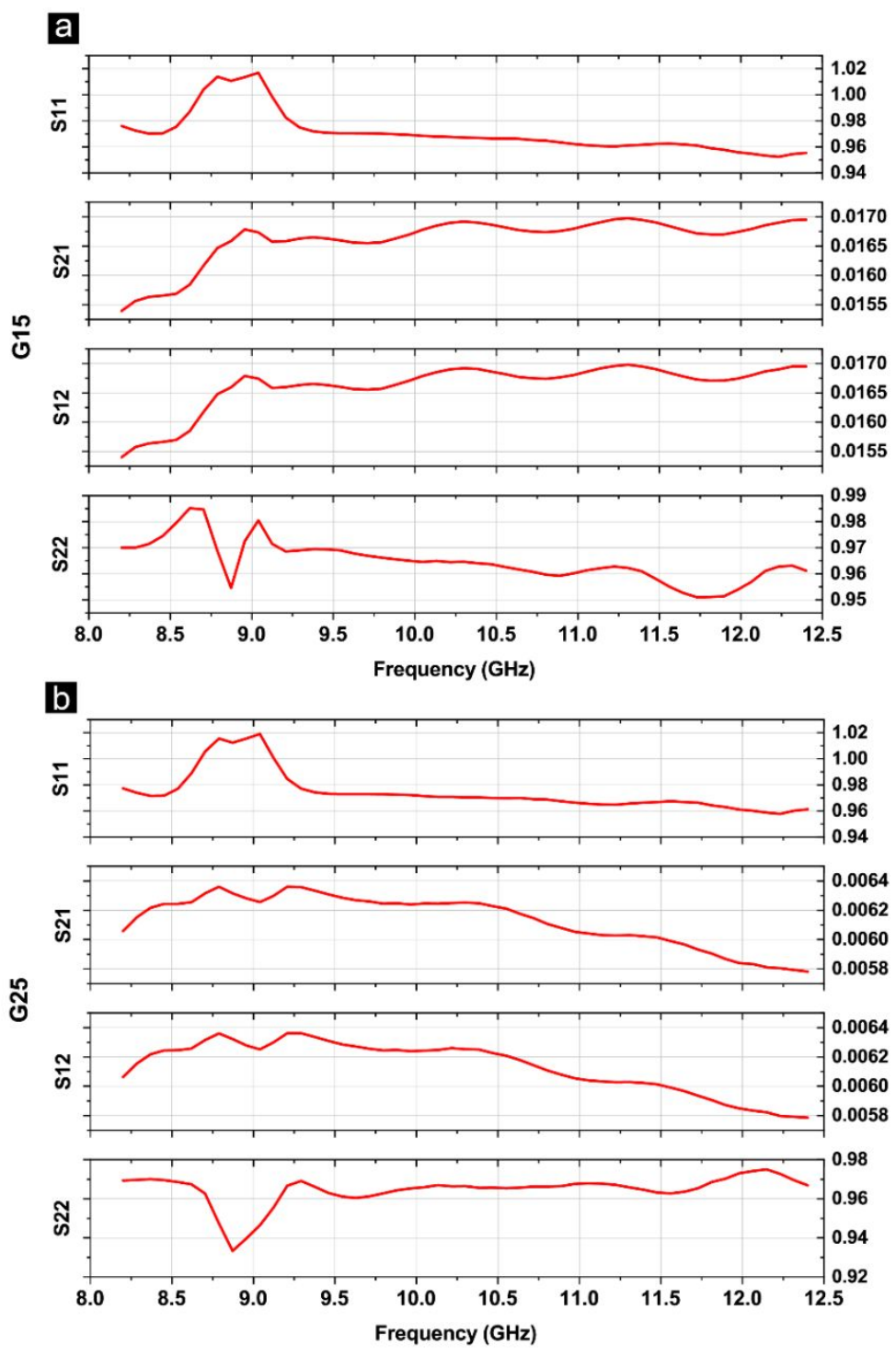

Figure S7. Magnitude of the S-paramters for (a) G15; and (b) G25 composites 
Cole-Cole diagrams for sintered pristine alumina and bulk composites with various GAIN content are presented in Figure S8. All samples show various polarization contributions represented by the multiple semicircles.

a

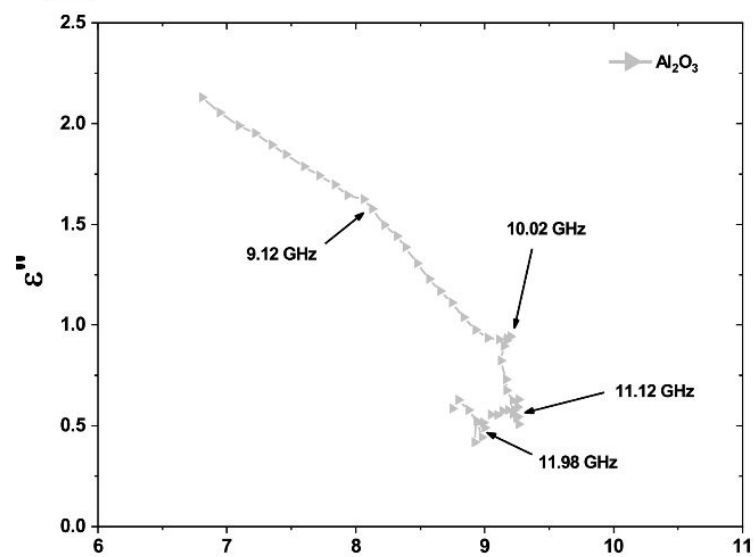

c
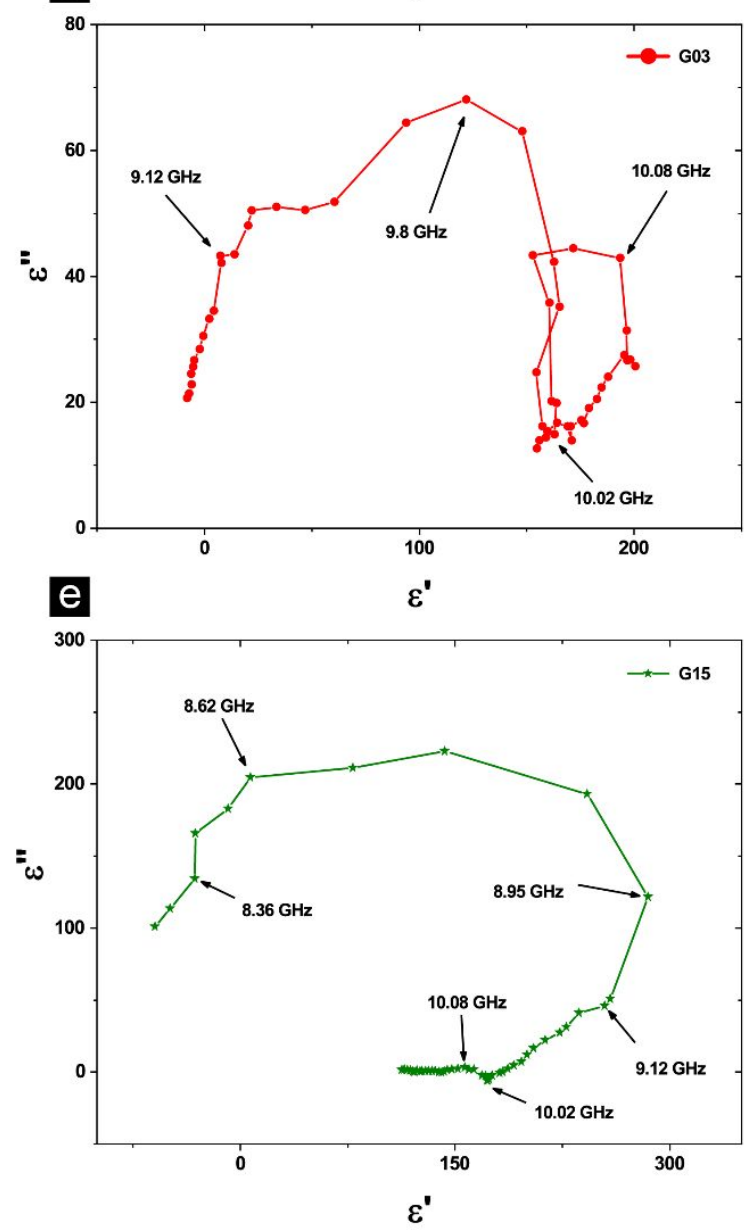

b

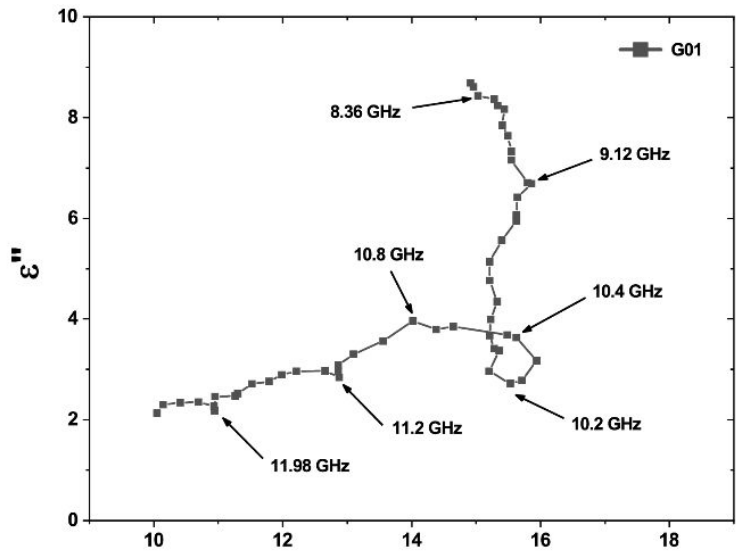

d
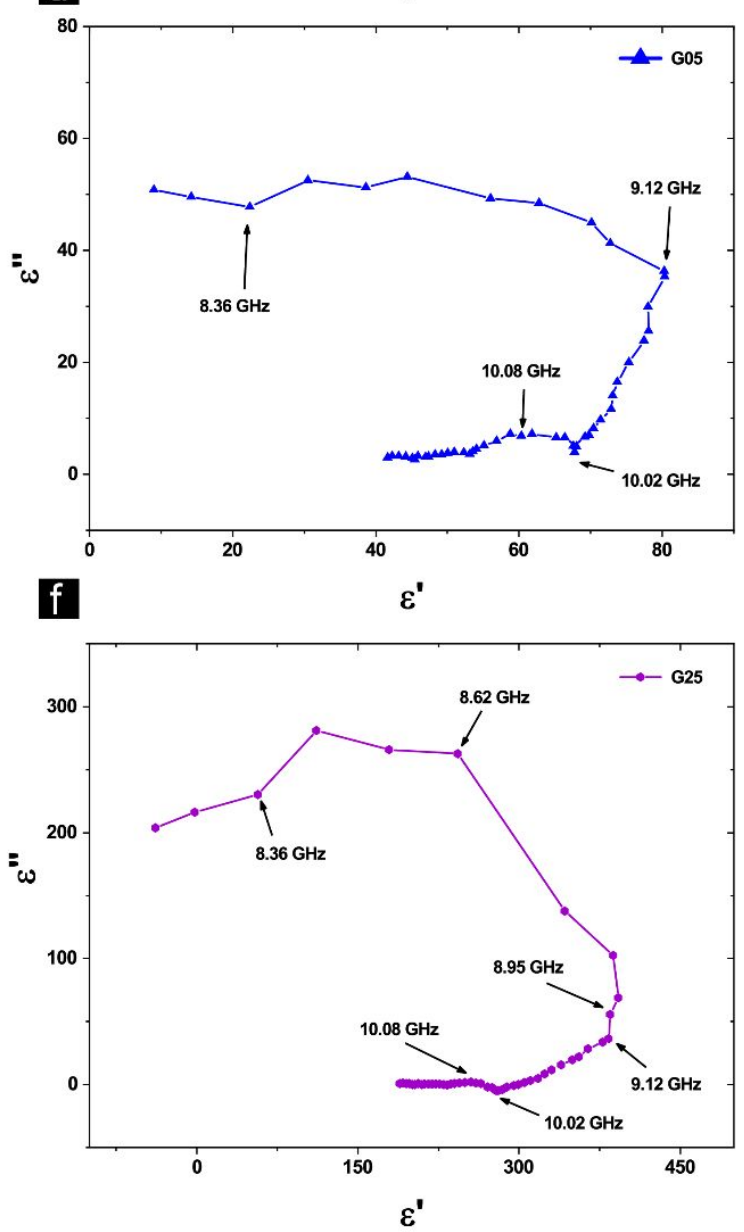

Figure S8. (a-f) Cole-Cole diagram of reference polycrystalline alumina and bulk composites as a function of GAIN loading from G01 to G25. 
For further optimization of absorption efficiency, several methods are proposed for designing multilayer absorbers based on impedance matching technique. Some of these methods are Spiral algorithm ${ }^{12}$, Fast pareto genetic algorithm ${ }^{13}$, and Wind driven optimization technique ${ }^{14}$. Monte Carlo simulation relies on random sampling in order to find accidental solutions to otherwise deterministic equations of impedance and surface reflection loss. In this work, utilizing Monte Carlo, the defined boundary conditions stipulated total thickness limitations, and $\mathrm{RL}<-10 \mathrm{~dB}$ while for each iteration $3 \times 10^{7}$ random layer thickness values were solved. Some examples of the findings are shown in Figure S9a. Solutions such as FSM-S1, and FSM-S3, provide near-full X-band absorption of more than 95\%. Coincidently, the same solutions have the best impedance matching as shown in Figure S9b. Further characterization is needed in order to investigate the experimental values of the proposed solutions.

a

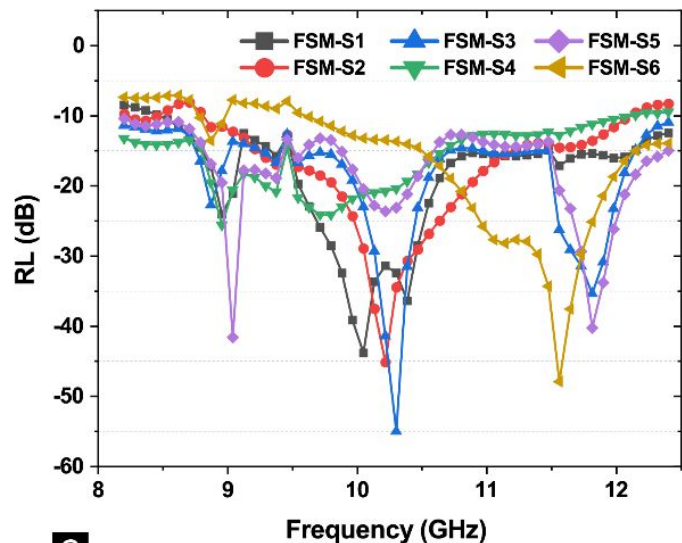

C

\section{b}

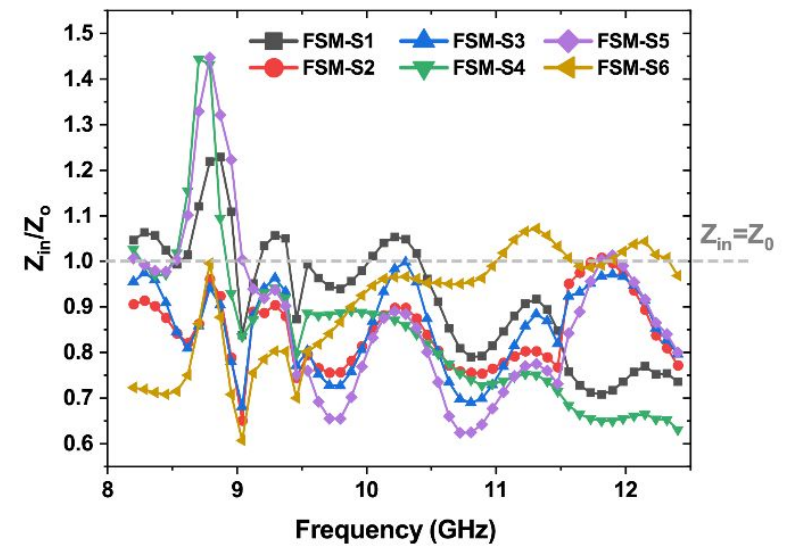

$n_{i-1} \sin \theta_{i-1}=n_{i} \sin \theta_{i}$

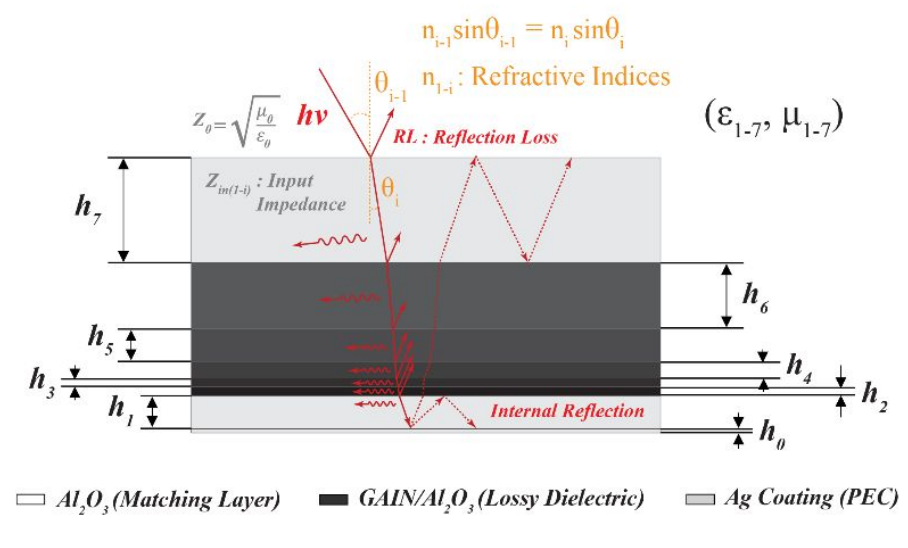

Figure S9. (a) Reflection loss vs frequency for absorber design solutions obtained from MonteCarlo approach to impedance matching; (b) normalized input impedance for the simulated solutions; and (c) the corresponding design parameters for the proposed solutions where $\mathrm{h}_{1}-\mathrm{h}_{7}$ are the thicknesses of the layers in micrometer and $h_{2}-h_{6}$ inversely correspond to G1-G25 composite structures. 


\section{References}

(1) Stankovich, S.; Dikin, D. A.; Piner, R. D.; Kohlhaas, K. A.; Kleinhammes, A.; Jia, Y.; Wu, Y.; Nguyen, S. T.; Ruoff, R. S. Synthesis of Graphene-Based Nanosheets via Chemical Reduction of Exfoliated Graphite Oxide. Carbon N. Y. 2007, 45 (7), 15581565. https://doi.org/10.1016/j.carbon.2007.02.034.

(2) Venezuela, P.; Lazzeri, M.; Mauri, F. Theory of Double-Resonant Raman Spectra in Graphene: Intensity and Line Shape of Defect-Induced and Two-Phonon Bands. Phys. Rev. B 2011, 84 (3), 035433. https://doi.org/10.1103/PhysRevB.84.035433.

(3) Eckmann, A.; Felten, A.; Mishchenko, A.; Britnell, L.; Krupke, R.; Novoselov, K. S.; Casiraghi, C. Probing the Nature of Defects in Graphene by Raman Spectroscopy. Nano Lett. 2012, 12 (8), 3925-3930. https://doi.org/10.1021/nl300901a.

(4) Ferrari, A. C. Raman Spectroscopy of Graphene and Graphite: Disorder, ElectronPhonon Coupling, Doping and Nonadiabatic Effects. Solid State Commun. 2007, 143 (1-2), 47-57. https://doi.org/10.1016/j.ssc.2007.03.052.

(5) Ferrari, A. C.; Basko, D. M. Raman Spectroscopy as a Versatile Tool for Studying the Properties of Graphene. Nat. Nanotechnol. 2013, 8 (4), 235-246.

https://doi.org/10.1038/nnano.2013.46.

(6) Tsoukleri, G.; Parthenios, J.; Papagelis, K.; Jalil, R.; Ferrari, A. C.; Geim, A. K.; Novoselov, K. S.; Galiotis, C. Subjecting a Graphene Monolayer to Tension and Compression. Small 2009, 5 (21), 2397-2402. https://doi.org/10.1002/smll.200900802.

(7) Stamatin, S. N.; Hussainova, I.; Ivanov, R.; Colavita, P. E. Quantifying Graphitic Edge Exposure in Graphene-Based Materials and Its Role in Oxygen Reduction Reactions. ACS Catal. 2016, 6 (8), 5215-5221. https://doi.org/10.1021/acscatal.6b00945.

(8) Saito, R.; Hofmann, M.; Dresselhaus, G.; Jorio, A.; Dresselhaus, M. S. Raman Spectroscopy of Graphene and Carbon Nanotubes. Adv. Phys. 2011, 60 (3), 413-550. https://doi.org/10.1080/00018732.2011.582251.

(9) Pisana, S.; Lazzeri, M.; Casiraghi, C.; Novoselov, K. S.; Geim, A. K.; Ferrari, A. C.; Mauri, F. Breakdown of the Adiabatic Born-Oppenheimer Approximation in Graphene. Nat. Mater. 2007, 6 (3), 198-201. https://doi.org/10.1038/nmat1846.

(10) Das, A.; Pisana, S.; Chakraborty, B.; Piscanec, S.; Saha, S. K.; Waghmare, U. V.; Novoselov, K. S.; Krishnamurthy, H. R.; Geim, A. K.; Ferrari, A. C.; Sood, A. K. 
Monitoring Dopants by Raman Scattering in an Electrochemically Top-Gated

Graphene Transistor. Nat. Nanotechnol. 2008, 3 (4), 210-215.

https://doi.org/10.1038/nnano.2008.67.

(11) Ferrari, A. C.; Robertson, J. Origin of the 1150-Cm-1 Raman Mode in Nanocrystalline Diamond. Phys. Rev. B 2001, 63 (12), 121405.

https://doi.org/10.1103/PhysRevB.63.121405.

(12) García, E.; Amaya, I.; Correa, R. Design of an Optimal Multilayer Electromagnetic Absorber through Spiral Algorithm. Ing. y Univ. 2015, 20 (1). https://doi.org/10.11144/javeriana.iyu20-1.dome.

(13) Weile, D. S.; Michielssen, E.; Goldberg, D. E. Genetic Algorithm Design of Pareto Optimal Broadband Microwave Absorbers. IEEE Trans. Electromagn. Compat. 1996, 38 (3), 518-525. https://doi.org/10.1109/15.536085.

(14) Ranjan, P.; Choubey, A.; Mahto, S. K. A Novel Approach for Optimal Design of Multilayer Wideband Microwave Absorber Using Wind Driven Optimization Technique. AEU - Int. J. Electron. Commun. 2018, 83, 81-87. https://doi.org/10.1016/j.aeue.2017.08.039. 